\title{
NONORTHOGONAL OPTIMAL PARTIAL DIALLEL CROSS DESIGNS FOR CONSISTENT ESTIMATION OF HERITABILITY
}

\author{
Himadri Ghosh*
}

\begin{abstract}
Diallel cross designs in the framework of random effects model are considered for the estimation of ratio of variance components, viz. heritability of crosses of inbred lines. New methods for construction of partial diallel cross (PDC) design sunder unblocked and blocked set up are proposed. The resulting designs of these methods are capable of minimizing variance of the estimator of heritability. Consistency of the estimator is also established. One of the practical advantage of the proposed series of designs is attributed to its ability to reduce number of distinct crosses over those for complete diallel cross (CDC) to an extent of 10 to 20 percent. Another heartening aspect of the proposed methods is that the blocks of these designs are incomplete and have smaller block sizes up to one-third than those of complete block designs. Note that, the variance-minimization criterion for optimality reduces to MS-optimality criterion of PDC designs defined in the context of fixed effects model. Further, the newly constructed designs are proved to be asymptotically universally optimal under fixed effects model. Since construction of these designs under blocked set up is quite involved, therefore a computer program is written in $\mathrm{C}++$ for generating these designs which is provided in the Appendix.
\end{abstract}

Key words and phrases: Efficient designs, heritability, optimal diallel cross designs, orthogonal-nonorthogonal blocking, partially balanced incomplete block design, variance components.

\section{Introduction}

Design of experiments for diallel crosses are used for studying genetic properties of inbred lines, known as general combining ability (g.c.a.) effects, in plant breeding experiments and has received considerable attention in the literature too, see e.g. Kempthorne and Curnow (1961), Hinkelmann (1975), Gupta et al. (1995), Ghosh and Das (2003a), Ghosh et al. (2005), and Singh et al. (2012). Random effects model are considered due to random selection of g.c.a. effects from a large hypothetical population of genetic component effects of inbred lines with variance $\sigma_{g}^{2}$ which is to be estimated to study genetic properties. Note that the number of possible crosses increases rapidly as the number of inbred lines increases. On the contrary, a small set of CDC is not capable of estimating population variance of g.c.a. effects since the estimator based on variance of a small number of observed lines would be subject to a large sampling error. Therefore, Kempthorne and Curnow (1961) considered PDC by incorporating a large number of inbred lines in the design through selection of only a subset

\footnotetext{
Received August 8, 2016. Revised December 6, 2016. Accepted April 13, 2017.

*Division of Statistical Genetics, ICAR-Indian Agricultural Statistics Research Institute, New Delhi110012, India. Email: hghosh@gmail.com
} 
from the set of all possible crosses. Note that, Griffing (1956) had earlier considered random effects of g.c.a., known as Model II, in the context of analysis of CDC. Our primary interest is to estimate relative magnitude of genetic variance components with respect to phenotypic variance. Since g.c.a. effects are assumed to be the only source of genetic component effects, it is of interest to obtain optimal and/or efficient designs for estimation of heritability $h^{2}$, which is defined as $h^{2}=4 \sigma_{g}^{2} /\left(2 \sigma_{g}^{2}+\sigma_{e}^{2}\right)$, where $4 \sigma_{g}^{2}$ and $\left(2 \sigma_{g}^{2}+\sigma_{e}^{2}\right)$ represent additive variance and phenotypic variance respectively, while $g$ and $e$ represent g.c.a. and random effect respectively. Let $T$ be an unbiased estimator of ratio of variance components $\sigma_{g}^{2} / \sigma_{e}^{2}$, then $4 T /(2 T+1)$ is an asymptotically unbiased estimator of $h^{2}$ and its asymptotic variance is proportional to variance of $T$. Hence, it is enough to study optimal and/or efficient designs, which minimize the variance of $T$ for all values of $\sigma_{g}^{2}$ and $\sigma_{e}^{2}$. Under fixed effects set up, Gupta and Kageyama (1994) introduced the method of constructing block designs for diallel crosses by treating lines rather than crosses as treatments. The authors also introduced the construction of optimal designs using nested balanced incomplete block designs. Some more series of optimal CDC designs were also constructed by Das et al. (1998a).

In this paper, our objective is to develop new methods of small size designs in the context of optimal and/or efficient estimation of heritability (Ghosh and Das (2003a), Ghosh et al. (2005)). We present novel methods of construction of designs in one replication of a PDC directly without resorting to the approach of Gupta and Kageyama (1994). Construction of our efficient series of designs is simpler than that of our optimal designs. The optimality problem is shown to be directly connected with MS-optimal diallel cross design under fixed effects model (Das et al. (1998b)). Note that, our criterion of optimality is different from that of usual $A, D, E$-optimality, etc. considered in fixed effects paradigm. However, efficiencies of these designs under fixed effects should be high by virtue of their asymptotic universal optimality property. To the best of our knowledge, no universally optimal PDC designs have so far been obtained.

The remaining part of the paper is organized as follows. In Section 2, we revisit unbiased estimation of $\sigma_{g}^{2} / \sigma_{e}^{2}$ for asymptotically unbiased estimation of $h^{2}$ (Ghosh et al. (2005)) and its optimality criterion. New series of optimal unblocked designs are obtained in Section 3. In Subsection 4.1, examples of blocked non-optimal PDC designs based on the method of treatment as crosses are discussed, which are shown to be not optimal. Therefore, in Subsection 4.2, necessary and sufficient condition of given relation between a pair of designs is derived to characterize series of efficient designs based on transitivity property of the relation. Two new methods of constructing blocked PDC designs are proposed. Optimal/efficient designs are characterized along with showing consistency of estimator $T$ by using eigen-values of $C$-matrix of new designs. Practical utility of these designs in terms of reduced number of crosses and block size as compared to that of orthogonal designing of CDC is also discussed. The designs have proved to be asymptotically universally optimal under fixed effects model. 
In Section 5, a series of optimal designs is constructed for some given class of designs. Although these designs are obtained in a restricted class, these are far more efficient than those obtained in Section 4. The performance of these designs in terms of consistent estimation of heritability and asymptotic universal optimality is also described. Finally, a computer program is written in $\mathrm{C}++$ to enable the plant breeders/geneticists to apply the methodology developed in the present article and is included as an Appendix.

\section{Some preliminaries}

Consider a diallel cross experiment using a design $d$ with $p$ lines and $b$ blocks each having $k$ crosses in modified or half-diallel and denoted by $\{i, j\}, i<j$ (see Singh and Hinkelmann (1995)). The model is

$$
\boldsymbol{Y}=\mu \mathbf{1}_{\nu}+D_{2}^{\prime} \boldsymbol{\beta}+D_{1}^{\prime} \boldsymbol{g}+\boldsymbol{e}
$$

where $\boldsymbol{Y}$ is the vector of $\nu(=b k)$ observations, $\mu$ is the general mean, $\boldsymbol{g}$ is the $p \times 1$ vector of g.c.a. effects with $E(\boldsymbol{g})=0$ and $\operatorname{Disp}(\boldsymbol{g})=\sigma_{g}^{2} I, \boldsymbol{\beta}$ is fixed effect due to blocks and $\boldsymbol{e}$ is error vector with $E(\boldsymbol{e})=0$ and $\operatorname{Disp}(\boldsymbol{e})=\sigma_{e}^{2} I$. Also, $D_{1}$ and $D_{2}$ are respectively the $p \times \nu$ line versus observation and $b \times \nu$ block versus observation matrices. Here $\mathbf{1}_{t}$ represents a $t \times 1$ column vector of all ones and $I_{t}$ denotes an identity matrix of order $t$. Let $G_{d}=D_{1} D_{1}^{\prime}=\left(g_{d i j}\right)$ and $\boldsymbol{s}_{d}=\left(s_{d 1}, s_{d 2}, \ldots, s_{d p}\right)^{\prime}=D_{1} \mathbf{1}$. Then $g_{d i j}$ stands for replication of cross $\{i, j\}$, $i \neq j$. Let $N_{d}=D_{1} D_{2}^{\prime}=\left(n_{d i j}\right)$ be the incidence matrix, where $n_{d i j}$ indicates the number of times the $i$ th line occurs in $j$ th block of design $d$. Let $C$-matrix of design $d$ be defined as $C_{d}=G_{d}-(1 / k) N_{d} N_{d}^{\prime}$. Note that, $C$-matrix of unblocked design $d$ is defined as $C_{0 d}=G_{d}-(1 / \nu) s_{d} s_{d}^{\prime}$.

Observed variance in $\boldsymbol{Y}$ denoted by $S S T$ may be split into the sum of squares due to lines after adjusting blocks (SSL), unadjusted blocks (SSB) and errors (SSE), based on Henderson's Method III, which gives $S S T=S S B+S S L+S S E$. Using independence between quadratic forms $S S L$ and $S S E$, Ghosh et al. (2005) proved the following theorem:

THEOREM 2.1. Let $d$ be a design with p lines, b blocks each of size $k$. Then, variance of the unbiased estimator

$$
T=\{(\nu-b-p-1)(S S L / S S E)-p+1\} / \operatorname{tr} C_{d}
$$

$$
\begin{aligned}
& \text { of } \sigma_{g}^{2} / \sigma_{e}^{2} \text { is }(2.2) \\
& V\left(T ; d, \sigma_{g}^{2} \sigma_{e}^{2}\right) \\
& =\alpha\left\{(\nu-b-p-1) \sigma_{g}^{4}\left(\operatorname{tr} C_{d}^{2} / \operatorname{tr}^{2} C_{d}\right)+2 t \sigma_{e}^{2} \sigma_{g}^{2}\left(1 / \operatorname{tr} C_{d}\right)\right. \\
& \left.+t(p-1) \sigma_{e}^{4}\left(1 / t r^{2} C_{d}\right)+\sigma_{g}^{4}\right\},
\end{aligned}
$$

where $\alpha=2 /\left\{(\nu-b-p-3) \sigma_{e}^{4}\right\}$ and $t=\nu-b-2$. 
Note that above result for unblocked diallel cross designs can be easily obtained by taking the number of blocks to be unity and $C$-matrix of the unblocked diallel cross design $d$ as $C_{0 d}$.

Let $\mathfrak{D}(p, \nu)$ be the class of unblocked diallel cross designs with $p$ lines and $\nu$ crosses, where $\mathfrak{D}_{0}(p, \nu)$ denotes the subclass of designs in $\mathfrak{D}(p, \nu)$ with $s_{d i}=2 \nu / p$; $i=1,2, \ldots, p$. For blocked diallel cross designs, let $\mathfrak{D}(p, b, k)$ be the class of designs with $p$ lines arranged in $b$ blocks of size $k$. Finally, let $\mathfrak{D}_{0}(p, b, k)$ be the subclass of designs in $\mathfrak{D}(p, b, k)$ for which $\operatorname{tr} C_{d}$ is maximum and $\mathfrak{D}_{0 e}(p, b, k)$ be the equi-replicated subclass of designs in $\mathfrak{D}_{0}(p, b, k)$ for which $s_{d i}=2 b k / p, i=$ $1,2, \ldots, p, d \in \mathfrak{D}_{0 e}(p, b, k)$. The following lemmas will be useful for characterizing optimal designs:

LEMmA 2.1 (Cheng (1978)). For given positive integers $q$ and $t$, the minimum of $r_{1}^{2}+r_{2}^{2}+\cdots+r_{q}^{2}$ subject to $r_{1}+r_{2}+\cdots+r_{q}=t$, where $r_{i}$ 's are non-negative integers, is obtained when $t-q[t / q]$ number of the $r_{i}$ 's are equal to $[t / q]+1$ and $q-t+q[t / q]$ number are equal to $[t / q]$, where $[z]$ denotes the largest integer not exceeding $z$.

LEMma 2.2 (Ghosh (2003a)). For any design $d \in \mathfrak{D}(p, \nu), \operatorname{tr} C_{0 d} \leq 2 \nu(p-$ 2)/p. Equality holds iff $s_{d i}=2 \nu / p, i=1,2, \ldots, p$.

Lemma 2.3 (Das et al. (1998a)). For any design $d \in \mathfrak{D}(p, b, k), \operatorname{tr} C_{d} \leq$ $k^{-1} b\{2 k(k-1-2 x)+p x(x+1)\}$, where $x=[2 k / p]$. Equality holds iff $n_{\text {dij }}=x$ or $x+1, i=1,2, \ldots, p, j=1,2, \ldots, b$. If $2 k<p$, then $x=0$ and in that case $\operatorname{tr} C_{d} \leq 2 b(k-1), d \in \mathfrak{D}(p, b, k)$.

LEMmA 2.4 (Ghosh (2003a)). Consider a real symmetric square matrix $A$ of order $m$ and rank $r$. Then $t r A^{2} \geq(1 / r) t r^{2} A$. Equality holds iff all the non-zero eigenvalues of $A$ are equal.

A design $d$ is said to be globally optimal if, among all designs in $\mathfrak{D}(p, b, k)(\mathfrak{D}(p, \nu)), d$ minimizes $V\left(T ; d, \sigma_{g}^{2}, \sigma_{e}^{2}\right)$ in $(2.2)$. Observe that the expression in (2.2) is of the form:

$$
\xi\left(\operatorname{tr} C_{d}^{2} / \operatorname{tr}^{2} C_{d}\right)+\gamma\left(1 / \operatorname{tr} C_{d}\right)+\delta\left(1 / t r^{2} C_{d}\right)+\eta
$$

which depends on parameters $\sigma_{g}^{2}$ and $\sigma_{e}^{2}$ and information matrix $C_{d}$. Note that, minimization of (2.2) may be carried out by minimizing $\operatorname{tr} C_{d}^{2} / \operatorname{tr}^{2} C_{d}$ and maximizing $\operatorname{tr} C_{d}$. To this end, finding optimal design is very difficult unless we consider some meaningful subclasses of global classes $\mathfrak{D}(p, \nu)$ and $\mathfrak{D}(p, b, k)$ for unblocked and blocked PDC experiments. Therefore, equi-replicated class of designs $\mathfrak{D}_{0}(p, \nu)$ is considered to find a new series of optimal PDC designs under unblocked diallel cross experiment. Although the above approach is successfully applied for obtaining optimal PDC designs under orthogonal blocking but for larger block size (Das et al. (1998b)), very few successes have so far been achieved to obtain non-orthogonal optimal PDC designs with smaller block size. 
To this end, Mukerjee (1997) showed that some series of $E$-optimal PDC designs are also $A$ - and $D$-efficient at the cost of large block size. Ghosh et al. (2006) studied efficiencies of a new series of designs with reduced block size by using sharper bounds of the functional for $A$ - and $D$-criteria. The efficiencies were found to be as good as that of Mukerjee's method. Using Lemmas 2.2-2.4, lower bound of efficiency of unblocked and blocked PDC designs is defined by

$$
e f f\left(d ; \sigma_{g}^{2}, \sigma_{e}^{2}\right)=\frac{\xi(1 /(p-1))+\gamma \min _{d}\left(1 / t r C_{d}\right)+\delta \min _{d}\left(1 / t r^{2} C_{d}\right)+\eta}{\xi\left(t r C_{d}^{2} / \operatorname{tr}^{2} C_{d}\right)+\gamma\left(1 / \operatorname{tr} C_{d}\right)+\delta\left(1 / \operatorname{tr}^{2} C_{d}\right)+\eta} .
$$

In case of blocked diallel cross design, note that given two blocked designs $d_{1}, d_{2} \in$ $\mathfrak{D}_{0}(p, b, k)$, eff $\left(d_{1} ; \sigma_{g}^{2}, \sigma_{e}^{2}\right) \leq$ eff $\left(d_{2} ; \sigma_{g}^{2}, \sigma_{e}^{2}\right) \leq 1$ (this is denoted by $\left.d_{2}>d_{1}\right)$ for all values of $\left(\sigma_{g}^{2}, \sigma_{e}^{2}\right)$ iff $\operatorname{tr} C_{d_{1}}^{2} \leq \operatorname{tr} C_{d_{2}}^{2}$. Now, given a block diallel cross design, eff $\left(d ; \sigma_{g}^{2} \sigma_{e}^{2}\right) \approx(1 /(p-1)) /\left(\operatorname{tr} C_{d}^{2} / \operatorname{tr}^{2} C_{d}\right) \approx 1$, iff $1 /(p-1) \approx \operatorname{tr} C_{d}^{2} / \operatorname{tr}^{2} c_{d}$. Here, the approximation notation $a \approx b$ stands for $a / b$ is close to unity. It may be mentioned that Ghosh et al. (2005) have given a series of efficient designs in the class $\mathfrak{D}_{0}(m n, n, m(n-1) / 2)$ by considering the above approximation and found that for $2 \leq m \leq 15$ and $5 \leq n \leq 15, n$ being odd, the efficiencies are at least 0.893 , where variance components vary in the domain $(0.1,3.0)$. For the present study also, this approximation holds true thereby yielding efficiencies close to unity, in view of Table 1. Further, construction of parameter dependent optimal or efficient design, i.e. $d\left(\sigma_{g}^{2}, \sigma_{e}^{2}\right)$ is meaningful only when the parameters $\sigma_{g}^{2}$ and $\sigma_{e}^{2}$ are assumed to be known, which hardly ever holds. In case of unblocked set up, a new series of optimal PDC designs are obtained in $\mathfrak{D}_{0}(p, \nu)$ whose efficiency is unity. It may be noted that above assumption does not pose a problem in this case as the optimal design $d_{0}^{*}=d\left(\sigma_{g}^{2}, \sigma_{e}^{2}\right)$ does not depend on $\left(\sigma_{g}^{2}, \sigma_{e}^{2}\right)$ and is characterized by using the following theorem of Ghosh and Das (2003a) on $A$-optimal designs under random effects model:

TheOrem 2.2. A design $d_{0}^{*}$ with $p$ lines is A-optimal in $\mathfrak{D}_{0}(p, n)$ with $s_{i}=$ $2 \nu / p=s$ iff the number of times $\left(g_{d_{0}^{*} i i^{\prime}}\right)$ that cross $\left\{i, i^{\prime}\right\}$ occurs in $d_{0}^{*}$ satisfies $\left|g_{d_{0}^{*} i i^{\prime}}-s /(p-1)\right|<1, i \neq i^{\prime}, i, i^{\prime}=1,2, \ldots, p$.

Using this theorem, and noting that $A$-optimal design $d_{0}^{*}=$ $\arg \min _{d \in \mathfrak{D}_{0}(p, n)} \operatorname{tr} C_{d}^{2}$; it is concluded that $d_{0}^{*}$ is also optimal in $\mathfrak{D}_{0}(p, \nu)$ for asymptotically unbiased estimation of heritability.

\section{Optimal PDC designs based on circular PBIB designs}

Analogous to circular design, Kempthorne and Curnow (1961) constructed PDC with $p$ lines and replication $2 s$; thus $\nu=p s$, where $p$ and $s$ both are not even. Here the number of distinct crosses is $p s / 2$. As per the authors' construction method, unblocked diallel cross design $d \in \mathfrak{D}(5,10)$ consisting of crosses, viz. $\{(1,3),(1,4),(2,4),(2,5),(3,5)(1,3),(1,4),(2,4),(2,5),(3,5)\}$ is obtained. Note that, this design is not optimal as the range of $g_{d i j}$ 's (i.e. $\max _{i j} g_{d i j}-\min _{i j} g_{d i j}$ ) is large by noting the fact that some cross $\{1,4\}$ say, occurs twice, i.e. $g_{d 1,4}=2$ while some other cross $\{1,5\}$ does not occur in the design, i.e. $g_{d 1,5}=0$. To eliminate 
this drawback, a new series of optimal PDC designs in $\mathfrak{D}_{0}((3 k-1), k(3 k-1) / 2)$ is obtained by us and the method of construction is as follows:

$$
\begin{aligned}
& S_{i}=\{(i, i+k+j): j=0,1, \ldots, k-1\}, \quad i=0,1, \ldots, k-1 \\
& S_{l}=\{(l, l+k+j): j=0,1, \ldots, 2 k-l-2\}, \quad l=k, k+1, \ldots,(2 k)-2 .
\end{aligned}
$$

Above crosses can be generated from association scheme of a circular design by considering blocks of sizes 2 and is described as follows: $j$ is $q$ th associate of $i$ iff $j=i \mp(q-1) \bmod (3 k-1)$, where $q=2,3, \ldots, 3 k / 2$, if $k$ is even and $q=2,3, \ldots,(3 k+1) / 2$, if $k$ is odd. When $k$ is even, only $k / 2$ associates, where each of $q$ th $(q=k+1, \ldots, 3(k / 2))$ associate of a symbol (line) has cardinality 2 with which the line is crossed $\lambda_{q}=1$ times. For other $(k-1)$ associates, $\lambda$ 's are 0 . When $k$ is odd, each of $q$ th $(q=k+1, \ldots,((3 k-1) / 2))$ associate of a symbol has cardinality 2 with which the line is crossed $\lambda_{q}=1$ times, whereas $((3 k+1) / 2)$ th associate of a symbol has cardinality 1 with which the line is crossed $\lambda_{((3 k+1) / 2)}=1$ times. For other $(k-1)$ associates, $\lambda$ 's are 0 .

Example 3.1. Suppose $k=3$. Then $p=8$ and $\nu=12$. Using our method of construction, following design:

$$
\{(0,3),(0,4),(0,5),(1,4),(1,5),(1,6),(2,5),(2,6),(2,7),(3,6),(3,7),(4,7)\}
$$

is obtained. It may be noted that the condition of Theorem 2.2 is satisfied since every line appears $s=3$ times giving $s /(p-1)=0.42$ and each cross $\{i, j\}$ appears $g_{d i j}$ times which can take values either 0 or 1 . Therefore, this design is optimal in $\mathfrak{D}_{0}(8,12)$.

\section{Construction of some efficient block designs}

\subsection{Motivation}

One of the ad-hoc methods for construction of PDC designs is to first obtain partially balanced incomplete block design (PBIBD) with block size 2 referred to as auxiliary (AUX) design. Using the correspondence between treatments and lines, and between blocks and crosses, AUX design determines the mating (M) design in the following way. The M-design is characterized by cross vs. line incidence matrix of AUX design. These crosses are to be grown by using appropriate incomplete block design, called environment (ENV) design (Singh and Hinkelmann (1988)). One possible approach to get this design is that the cross vs. line incidence matrix of M-design may be used to obtain treatment (cross) vs. block incidence matrix of the ENV design and the resultant design is called embedded mating design into environment (M-ENV) design.

Example 4.1. This example, cited in Singh and Hinkelmann (1995), is demonstrated to show that the resultant M-ENV design could neither maximize $\operatorname{tr} C$ nor minimize $\operatorname{tr} C^{2}$. This is due to the fact that it yields large values of ranges for each $n_{d i j}$ 's (i.e. $\max _{i} n_{d i j}-\min _{i} n_{d i j}$, for each $j$ ) and $g_{d i j}$ 's 
(i.e. $\max _{i j} g_{d i j}-\min _{i j} g_{d i j}$ ) respectively. To this end, authors used conventional PBIBD to obtain AUX-design by using triangular association scheme with $p$ treatments each replicated $s$ times arranged in $b$ blocks of size $k$, and considered following values viz. $p=10, b=15, s=3, k=2, \lambda_{1}=0, \lambda_{2}=1$ for selecting the PDCs. Note that the number of first and second associates are $n_{1}=6$ and $n_{2}=3$ respectively. Following the above two-step procedure of selecting crosses (AUX-design) and arranging the crosses in blocks (ENV-design) using M-design, embedded M-ENV-1 design is obtained which is given below:

\begin{tabular}{llll}
\hline Block I & $(1,8)$ & $(1,9)$ & $(1,10)$ \\
Block II & $(2,6)$ & $(2,7)$ & $(2,10)$ \\
Block III & $(3,5)$ & $(3,7)$ & $(3,9)$ \\
Block IV & $(4,5)$ & $(4,6)$ & $(4,8)$ \\
Block V & $(3,5)$ & $(4,5)$ & $(5,10)$ \\
Block VI & $(2,6)$ & $(4,6)$ & $(6,9)$ \\
Block VII & $(2,7)$ & $(3,7)$ & $(7,8)$ \\
Block VIII & $(1,8)$ & $(4,8)$ & $(7,8)$ \\
Block IX & $(1,9)$ & $(3,9)$ & $(6,9)$ \\
Block X & $(1,10)$ & $(2,10)$ & $(5,10)$ \\
\hline
\end{tabular}

Note that this design has few number of distinct crosses with fewer number of lines within a block, thereby giving large values of ranges for $g_{d i j}$ 's and $n_{d i j}$ 's, which reduces the design efficiency. For the above design $d \in D(10,10,3)$, it is observed that $\operatorname{tr} C=20$, which is much smaller than its upper bound of 40 . Note that a series of designs can be described in general for a triangular association scheme of $n(n-1) / 2$ treatments by making crosses as pairs of 2 nd associates.

Example 4.2. The purpose of this example is to give a motivation to construct an efficient and optimal PDC designs. Specifically, two designs are shown here to describe the method of construction of these designs by treating lines rather than crosses as treatments used in Example 4.1, and thereby enabling us to maximize $\operatorname{tr} C$ and minimize $\operatorname{tr} C^{2}$ in a given class of designs. Here, we used two-associate group divisible scheme to obtain the AUX-design for selecting PDCs. Specifically, in our case $p=8, b=24, s=6, k=2 ; \lambda_{1}=0, \lambda_{2}=1$. The procedure of embedding M-design in an ENV-design is carried out by using another PBIBD design using two associate group divisible scheme with following values viz. $p=b=8, s=k=3, \lambda_{1}=0, \lambda_{2}=1$ and $m=4, n=2$. The incomplete blocks of the PBIBD may be delineated as follows:

$\begin{array}{llll}\text { Block I } & 1 & 2 & 3 \\ \text { Block II } & 4 & 5 & 6 \\ \text { Block III } & 1 & 4 & 7 \\ \text { Block IV } & 2 & 5 & 8\end{array}$


where the groups are represented as

\begin{tabular}{llll} 
Block V & 1 & 6 & 8 \\
Block VI & 3 & 5 & 7 \\
Block VII & 2 & 6 & 7 \\
Block VIII & 3 & 4 & 8 \\
\hline
\end{tabular}

\begin{tabular}{lll}
\hline Group I & 1 & 5 \\
Group II & 2 & 4 \\
Group III & 3 & 6 \\
Group IV & 7 & 8 \\
\hline
\end{tabular}

Note that the number of first and second associates are $n_{1}=1$ and $n_{2}=6$ respectively. The M-ENV-2 design is obtained by identifying crosses with blocks of AUX design arranged in 8 blocks of PBIBD. The occurrence of cross $(i, j)$ in a block is 1 or 0 whenever both treatments $i$ and $j$ appear or do not appear in the same block of PBIBD respectively. The resultant M-ENV-2 design is given below:

\begin{tabular}{llll}
\hline Block I & $(1,2)$ & $(1,3)$ & $(2,3)$ \\
Block II & $(4,5)$ & $(4,6)$ & $(5,6)$ \\
Block III & $(1,4)$ & $(1,7)$ & $(4,7)$ \\
Block IV & $(2,5)$ & $(2,8)$ & $(5,8)$ \\
Block V & $(1,6)$ & $(1,8)$ & $(6,8)$ \\
Block VI & $(3,5)$ & $(3,7)$ & $(5,7)$ \\
Block VII & $(2,6)$ & $(2,7)$ & $(6,7)$ \\
Block VIII & $(3,4)$ & $(3,8)$ & $(4,8)$ \\
\hline
\end{tabular}

However, one drawback of our methodology is that the number of times that each line appears in a block are scattered i.e. $\max _{i} n_{d i j}-\min _{i} n_{d i j}=2$, implying thereby that it is not possible to maximize $\operatorname{tr} C$. For the above design $d \in D(8,8,3)$, it is observed that $\operatorname{tr} C=16$, which is much smaller than its upper bound of 32. To remove this drawback, a possible better design may be obtained by rearranging the crosses across blocks where $\operatorname{tr} C$ attains its maximum and value of $\operatorname{tr} C^{2} / t r^{2} C$ is 0.14 , which is close to its lower bound. Thus the modified PDC design is as follows:

\begin{tabular}{llll}
\hline Block I & $(1,2)$ & $(3,4)$ & $(6,7)$ \\
Block II & $(3,7)$ & $(1,6)$ & $(2,8)$ \\
Block III & $(1,4)$ & $(5,7)$ & $(2,6)$ \\
Block IV & $(2,5)$ & $(4,7)$ & $(3,8)$ \\
Block V & $(4,6)$ & $(2,7)$ & $(5,8)$ \\
Block VI & $(3,5)$ & $(4,8)$ & $(1,7)$ \\
Block VII & $(6,8)$ & $(1,3)$ & $(4,5)$ \\
Block VIII & $(2,3)$ & $(5,6)$ & $(1,8)$ \\
\hline
\end{tabular}


It may be pointed that the rearrangement of crosses of AUX design, i.e. pairs of 2nd associates, is very difficult to generalize. This observation is the genesis of general construction approach of arranging crosses as pairs of 2 nd associates for obtaining efficient non-orthogonal PDC designs.

\subsection{Efficient designs}

Consider $\mathfrak{D}_{0 e}(m n, m n,(m-1) n / 2)$ defined in Section 1. Note that, by Lemma $2.3, n_{d, i j}=0$ or 1 for any $d \in \mathfrak{D}_{0 e}(m n, m n,(m-1) n / 2)$. Define $X_{d}=\left(\left(x_{d, i i^{\prime}}\right)\right)$ where $x_{d, i i^{\prime}}$ denotes number of blocks in which both the lines $i$ and $i^{\prime}$ do not appear, $i \leq i^{\prime}$. Here, $x_{d, i i}=n$. The following theorem, which gives some direction to search series of efficient designs, establishes necessary and sufficient condition of transitive relation between pair-wise designs.

THEOREM 4.1. Let $m=2 t+1, t \geq 1, p=m n, n \geq 1, k=(m-1) n / 2$. Consider two designs $d_{1}$ and $d_{2} \in \mathfrak{D}_{0 e}(m n, m n,(m-1) n / 2)$, where $x_{d_{1}, i i^{\prime}}=$ $x_{d_{2}, i i^{\prime}}, i, i^{\prime}=1,2, \ldots, m n$. Then $d_{2}>d_{1}$, equivalently, $\operatorname{tr} C_{d_{2}}^{2} \leq \operatorname{tr} C_{d_{1}}^{2}$ iff

$$
\max _{\left(i, i^{\prime}\right) \neq\left(j, j^{\prime}\right): i<i^{\prime}, j<j^{\prime}}\left|g_{d_{2}, i i^{\prime}}-g_{2, j j^{\prime}}\right| \leq \max _{\left(i, i^{\prime}\right) \neq\left(j, j^{\prime}\right): i<i^{\prime}, j<j^{\prime}}\left|g_{d_{1}, i i^{\prime}}-g_{d_{1} j j^{\prime}}\right| .
$$

In other words, $d_{2}>d_{1}$ when range for replications $\left(g_{d, i j}\right)$ of crosses in design $d_{2}$ are less than those in design $d_{1}$.

Proof. Note that, $\operatorname{tr} C_{d}^{2}=\operatorname{tr} G_{d}^{2}-(2 / k) \operatorname{tr} G_{d} N_{d} N_{d}^{\prime}+\left(1 / k^{2}\right) \operatorname{tr} N_{d} N_{d}^{\prime} N_{d} N_{d}^{\prime}$. Let $N_{d} N_{d}^{\prime}=\left(\left(n_{d, i i^{\prime}}^{(2)}\right)\right)$. By usual set theoretic operation, and due to binary property of design $d$ :

$$
n_{d, i i^{\prime}}^{(2)}=m n-\left[\#\left\{k: n_{d, i k}=0\right\}+\#\left\{k: n_{d, i^{\prime} k}=0\right\}-x_{d, i i^{\prime}}\right]=x_{d, i i^{\prime}}+(m-2) n .
$$

Using the fact that $\mathbf{1}_{p}^{\prime} G_{d}=2(m-1) n \mathbf{1}_{p}^{\prime}$, we get

$$
\operatorname{tr} G_{d} N_{d} N_{d}^{\prime}=\operatorname{tr} G_{d}\left[n(m-2) \mathbf{1}_{p} 1_{p}^{\prime}+X_{d}\right]=4 k(m-2) p n+\operatorname{tr} G_{d} X_{d} .
$$

Define $N_{d}^{c}=\left(\left(n_{d, i j}^{c}\right)\right)$, where $n_{d, i j}^{c}=1$ or 0 according as $n_{d, i j}=0$ or 1 . Then

$$
\operatorname{tr} \mathbf{1}_{p} \mathbf{1}_{p}^{\prime} X_{d}=\operatorname{tr} \mathbf{1}_{p}^{\prime} X_{d} \mathbf{1}_{p}=\mathbf{1}_{p}^{\prime} N_{d}^{c} N_{d}^{c^{\prime}} \mathbf{1}_{p}=p(p-2 k)^{2}
$$

Using (4.2), we get

$$
\begin{aligned}
\operatorname{tr} N_{d} N_{d}^{\prime} N_{d} N_{d}^{\prime} & =\operatorname{tr}\left[n(m-2) \mathbf{1}_{p} \mathbf{1}_{p}^{\prime}+X_{d}\right]^{2} \\
& =(p n(m-2))^{2}+2(m-2) n \operatorname{tr} \mathbf{1}_{p} \mathbf{1}_{p}^{\prime} X_{d}+\operatorname{tr} X_{d}^{2} .
\end{aligned}
$$

By (4.1) and (4.3),

$$
\begin{aligned}
\operatorname{tr} C_{d}^{2}= & \operatorname{tr} G_{d}^{2}-(2 / k) \operatorname{tr} G_{d} X_{d}+\left(1 / k^{2}\right) \operatorname{tr} X_{d}^{2}-8(m-2) p n \\
& +\left(1 / k^{2}\right)\left\{(p n(m-2))^{2}+2(m-2) n p(p-2 k)^{2}\right\} \\
= & \operatorname{tr}\left[G_{d}-(1 / k) X_{d}\right]^{2}-8(m-2) p n \\
& +\left(1 / k^{2}\right)\left\{(p n(m-2))^{2}+2(m-2) n p(p-2 k)^{2}\right\} .
\end{aligned}
$$


Thus, it is enough to show that $\operatorname{tr}\left[G_{d_{1}}-(1 / k) X_{d_{1}}\right]^{2} \leq \operatorname{tr}\left[G_{d_{2}}-(1 / k) X_{d_{2}}\right]^{2}$ subject to $\sum_{i<i^{\prime}} g_{d, i i^{\prime}}=m(m-1) n^{2} / 2$, where

$$
\operatorname{tr}\left[G_{d}-(1 / k) X_{d}\right]^{2}=2 \sum_{i<i^{\prime}}\left\{g_{d, i i^{\prime}}-x_{d, i i^{\prime}} / k\right\}^{2}+p\left\{\left(\frac{(m-1)^{2} n-2}{(m-1)}\right)^{2}\right\}
$$

It can be here assumed that $g_{d, i i^{\prime}}-g_{d, j j^{\prime}} \leq-2$. Consider a hypothetical design $d^{*}$ and the functional $\operatorname{tr}\left[G_{d^{*}}-(1 / k) X_{d^{*}}\right]^{2}$, where $g_{d^{*}, i i^{\prime}}=g_{d, i i^{\prime}}+1$ and $g_{d^{*}, j j^{\prime}}=$ $g_{d, j j^{\prime}}-1$. Note that $x_{d, i i^{\prime}}=x_{d^{*}, i i^{\prime}}$. Then

$$
\begin{aligned}
& \operatorname{tr}\left[G_{d^{*}}-(1 / k) X_{d^{*}}\right]^{2}-\operatorname{tr}\left[G_{d}-(1 / k) X_{d}\right]^{2} \\
& \quad=4\left\{\left(g_{d, i i^{\prime}}-g_{d, j j^{\prime}}\right)+\left(x_{d, j j^{\prime}} / k-x_{d, i i^{\prime}} / k\right)+1\right\} .
\end{aligned}
$$

Since $m \geq 3$, we observe that $0 \leq x_{d, i i^{\prime}} / k \leq 2 /(m-1) \leq 1$; so $x_{d, j j^{\prime}} / k-x_{d, i i^{\prime}} / k \leq$ $2 /(m-1) \leq 1$ and using $g_{d, i i^{\prime}}-g_{d, j j^{\prime}} \leq-2$, we obtain $\left\{\left(g_{d, i i^{\prime}}-g_{d, j j^{\prime}}\right)+\left(x_{d, j j^{\prime}} / k-\right.\right.$ $\left.\left.x_{d, i i^{\prime}} / k\right)+1\right\} \leq 0$. The proof is complete by noting that one can always reach design $d_{2}$ from design $d_{1}$ in finite number of steps where in each step the functional $\operatorname{tr} C_{d}^{2}$ keeps decreasing. Finally, it is easy to note that the relation $d_{2}>d_{1}$ is transitive.

Remark 4.1. Using the fact that $\sum_{i<i^{\prime}} g_{d, i i^{\prime}}=m(m-1) n^{2} / 2$ for $d \in$ $\mathfrak{D}_{0 e}(m n, m n,(m-1) n / 2)$ and $\#\left\{\left(i, i^{\prime}\right): 1 \leq i<i^{\prime}<m n\right\}=\left(\begin{array}{c}m n \\ 2\end{array}\right)>m(m-$ 1) $n^{2} / 2$, a series of designs $\left\{d_{e}(m, n)\right\}$ may be constructed with $g_{d, i i^{\prime}}=0$ or 1 and study their efficiencies. For ease in presentation, let $p=m n$ lines be partitioned into $m$ sets of lines denoted by $Q_{j}$, where $Q_{j}=\left\{a_{u}^{j}: 0 \leq u \leq(n-1)\right\}$, $0 \leq j \leq(m-1)$. Note line $a_{u}^{j}$ represents line labelled as $n j+u+1$. The general method of construction of design may be carried out in two steps:

(i) Consider a randomized block design with $n$ treatments arranged in $m$ blocks, which may be symbolically represented as:

\begin{tabular}{cccc}
\hline $0_{0}$ & $1_{0}$ & $\cdots$ & $(n-1)_{0}$ \\
$0_{1}$ & $1_{1}$ & $\cdots$ & $(n-1)_{1}$ \\
$\cdots$ & $\cdots$ & $\cdots$ & $\cdots$ \\
$0_{(m-1)}$ & $1_{(m-1)}$ & & $(n-1)_{(m-1)}$ \\
\hline
\end{tabular}

(ii) For every occurrence of treatment $u, 0 \leq u \leq n-1$ and at its $l$-th replication, $0 \leq l \leq m-1$, i.e. $u_{l}$, replace treatment $u_{l}$ by a block $B_{l, u}$, say, consisting of $(m-1) n / 2$ crosses of the form $\left\{\left(a_{k}^{j+l}, a_{u+k}^{m-j+l}\right): 1 \leq j \leq(m-1) / 2,0 \leq k \leq\right.$ $(n-1)\}$, where $j+l, m-j+l$ reduced $\bmod (m)$ and $u+k$ reduced $\bmod (n)$. Note that, for a fixed treatment $u$, one can generate $m$ blocks of $d_{e}(m, n)$ with respect to running of symbol $l$, where each $l$ determines a class $\mathcal{C}_{l}$ of $(m-1)$ sets of lines given by $\mathcal{C}_{l}=\left\{Q_{k}: k \in\{j+l, m-j+l ; 1 \leq j \leq\right.$ $(m-1) / 2\}\}$. Further, contents of block $B_{l, u}$ of size $(m-1) n / 2$ are obtained by crossing lines in $(j+l)$ th set, i.e. $a_{k}^{j+l}$ with lines in $(m-j+l)$ th set shifted by $u$ positions, i.e. $a_{u+k}^{m-j+l}$. To obtain $m n$ blocks of design $d_{e}(m, n)$ 
this procedure is applied to all the $n$ treatments defined in Step (i), where each treatment will generate $m$ blocks with respect to running of symbol $l$ of $\mathcal{C}_{l}$, each of size $(m-1) n / 2$. Note that $d_{e}(m, n) \in \mathfrak{D}_{0 e}(m n, m n,(m-1) n / 2)$.

Example 4.3. As an illustration of the above method of construction, consider the following design with parameters $m=3, n=5$; so $p=b=15$, and $k=5$ where $g_{d, i i^{\prime}}=0$ or 1 :

\begin{tabular}{llllll}
\hline Block I & $(6,11)$ & $(7,12)$ & $(8,13)$ & $(9,14)$ & $(10,15)$ \\
Block II & $(6,12)$ & $(7,13)$ & $(8,14)$ & $(9,15)$ & $(10,11)$ \\
Block III & $(6,13)$ & $(7,14)$ & $(8,15)$ & $(9,11)$ & $(10,12)$ \\
Block IV & $(6,14)$ & $(7,15)$ & $(8,11)$ & $(9,12)$ & $(10,13)$ \\
Block V & $(6,15)$ & $(7,11)$ & $(8,12)$ & $(9,13)$ & $(10,14)$ \\
Block VI & $(1,11)$ & $(2,12)$ & $(3,13)$ & $(4,14)$ & $(5,15)$ \\
Block VII & $(1,12)$ & $(2,13)$ & $(3,14)$ & $(4,15)$ & $(5,11)$ \\
Block VIII & $(1,13)$ & $(2,14)$ & $(3,15)$ & $(4,11)$ & $(5,12)$ \\
Block IX & $(1,14)$ & $(2,15)$ & $(3,11)$ & $(4,12)$ & $(5,13)$ \\
Block X & $(1,15)$ & $(2,11)$ & $(3,12)$ & $(4,13)$ & $(5,14)$ \\
Block XI & $(1,6)$ & $(2,7)$ & $(3,8)$ & $(4,9)$ & $(5,10)$ \\
Block XII & $(1,7)$ & $(2,8)$ & $(3,9)$ & $(4,10)$ & $(5,6)$ \\
Block XIII & $(1,8)$ & $(2,9)$ & $(3,10)$ & $(4,6)$ & $(5,7)$ \\
Block XIV & $(1,9)$ & $(2,10)$ & $(3,6)$ & $(4,7)$ & $(5,8)$ \\
Block XV & $(1,10)$ & $(2,6)$ & $(3,7)$ & $(4,8)$ & $(5,9)$ \\
\hline
\end{tabular}

Remark 4.2. The efficiencies are calculated for $m=2 t+1 ; t=1, \ldots, 8$ and $n=2,3, \ldots, 15$ and are reported in Table 1. It is found that lower bounds of eff $\left(d_{e}(m, n)\right)$ are more than $95 \%$ when $t \geq 2, n \geq 5$. Since maximum value of efficiency is unity, therefore designs $d_{e}(m, n)$ are optimal when $t \geq 6, n \geq 7$ and $t \geq 9, n \geq 3$. Further, these designs are MS-optimal under fixed effects model. Also one can achieve the proportion of reduction of number of distinct crosses in PDC with respect to $\mathrm{CDC}$ to $(n-1) /(m n-1)$, i.e. between 10 to 20 percent for $2 \leq t \leq 4, n \geq 7$ with efficiencies more than $95 \%$. It may be noted that reduction of more than 10 percent is large in absolute terms when number of complete crosses is large. Further, blocks of these designs are incomplete having smaller block sizes up to 33 percent than those for complete block.

Remark 4.3. Let for a design $d, \lambda_{d 1} \leq \lambda_{d 2} \leq \cdots \leq \lambda_{d(p-1)}$ be the non-zero eigenvalues of $C_{d}$. Then after some algebra, we see that for $i=1,2, \ldots,(n-1) m$, $\lambda_{d_{e}(m, n) i}=\theta_{1}$ and for $i=(n-1) m+1, \ldots, m n-1, \lambda_{d_{e}(m, n) i}=\theta_{2}$ where $\theta_{1}=(m-1) n$ and $\theta_{2}=\theta_{1}-\{(m+1) / n\} /(m-1)$. Given, $n$ finite, note that $\theta_{1} / \theta_{2} \rightarrow 1$ as $m \rightarrow \infty$. This shows that $C_{d_{e}(m, n)}$ is completely symmetric 


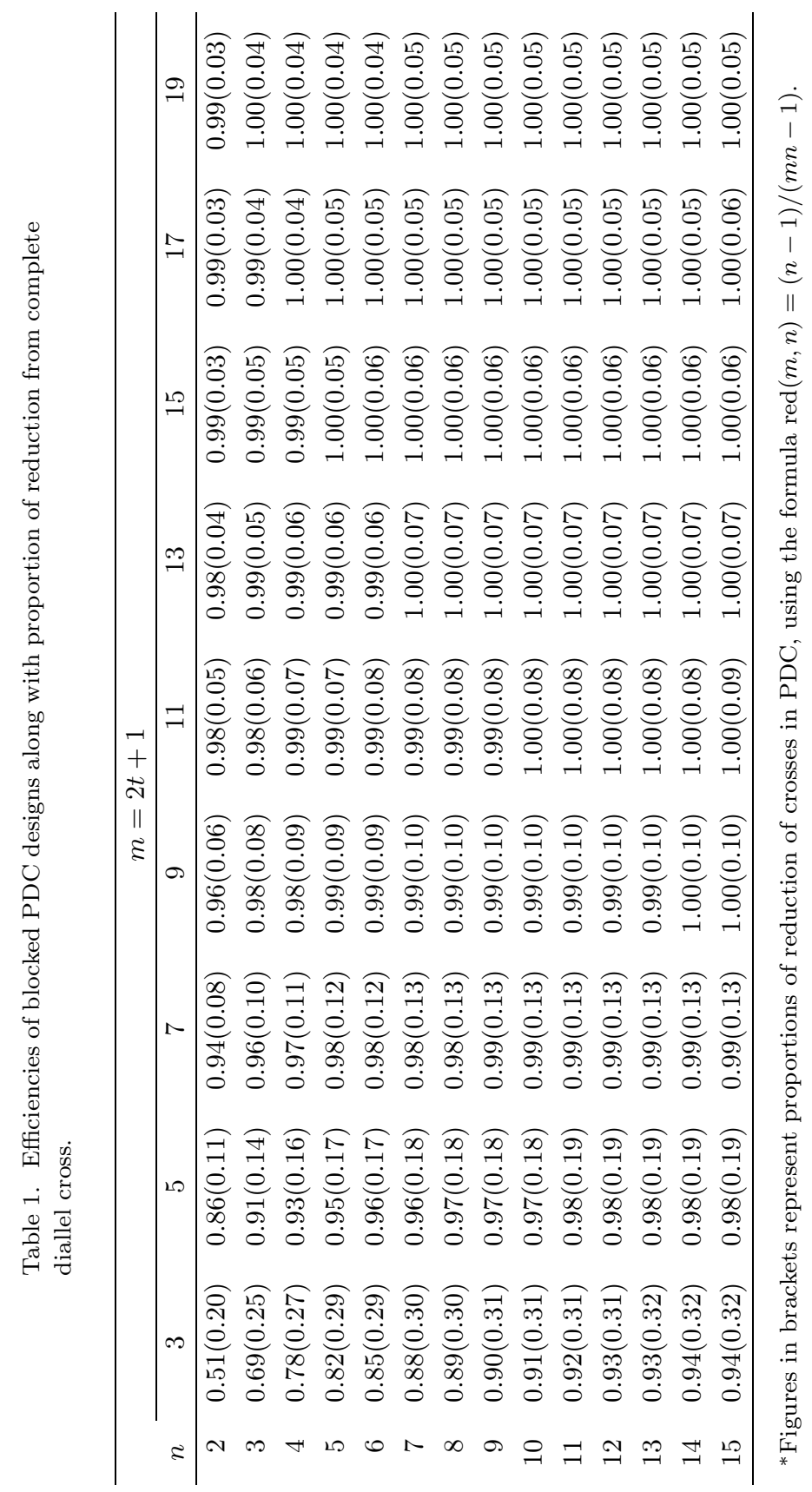


and has maximum trace, thus proving that the series of designs $d_{e}(m, n)$ are asymptotically universally optimal under fixed effects. Therefore, these designs should also have high efficiencies in the context of $A, D, E$-optimality under fixed effects model and $L$-optimality under random effects model (Ghosh and $\operatorname{Das}(2003 b))$.

Remark 4.4. Further, using Lemma $2.3\left(\operatorname{tr} C_{d} \leq 2 b(k-1)\right)$ and the fact $\sum_{i=1}^{p-1}\left(\lambda_{d i}-\bar{\lambda}\right)^{2}=0\left(m n^{2}\right)$, it can be shown that, eff $\left(d_{e}(m, n)\right) \rightarrow 1$ as $m, n \rightarrow$ $\infty$ which implies the series of designs $d_{e}(m, n)$ are asymptotically optimal and asymptotically MS-optimal under fixed effects model.

Remark 4.5. Note that, from $(2.2), \operatorname{t\alpha }\left(1 / \operatorname{tr} C_{d}\right) \approx 1 /(m n)^{2} \rightarrow 0$ as $m, n \rightarrow$ $\infty$, and $t \alpha(p-1)\left(1 / t r^{2} C_{d}\right) \approx 1 /(m n)^{3} \rightarrow 0$ as $m, n \rightarrow \infty$. Finally, using Remark 4.3, it is easy to show that $(\nu-b-p-1) \alpha\left(\operatorname{tr} C_{d}^{2} / \operatorname{tr}^{2} C_{d}\right) \approx 1 /(m n) \rightarrow$ 0 as $m, n \rightarrow \infty$. Here, $a_{n} \approx b_{n}$ implies $a_{n} / b_{n} \rightarrow 1$ as $n \rightarrow \infty$. Therefore, $V\left(T ; d_{e}(m, n), \sigma_{g}^{2}, \sigma_{e}^{2}\right) \rightarrow 0$ as $m, n \rightarrow \infty$, which shows that the series of designs $d_{e}(m, n)$ are useful for providing a consistent estimator of heritability.

\section{Construction of optimal blocked partial diallel cross designs}

Consider arrangement of labels of $m n$ blocks of a design in $\mathfrak{D}_{0 e}(m n, m n,(m-$ 1) $n / 2$ ) into $m$ sets, each set consisting of $n$ labels. Define a "class of sets (unique up to permutation) of labels of blocks" by $\mathcal{S}=\left\{S_{w}: S_{w}=\left\{i_{n w}, i_{n w+1}, \ldots\right.\right.$, $\left.i_{n(w+1)-1}\right\} ; i_{n w+j} \neq i_{n w^{\prime}+j^{\prime}},(w, j) \neq\left(w^{\prime}, j^{\prime}\right), w=0,1,2, \ldots,(m-1) ; 1=i_{0}<$ $\left.i_{n}<i_{2 n}<\cdots<i_{n(m-1)}=n(m-1)+1\right\}$. Consider a subclass $\mathfrak{D}_{0 e}^{*}(m n, m n,(m-$ 1)n/2) $\subset \mathfrak{D}_{0 e}(m n, m n,(m-1) n / 2)$, such that for any design $d \in \mathfrak{D}_{0 e}^{*}(p, b, k)$, there exists a class $\mathcal{S}_{d}$, say, (i.e. class of sets of labels of blocks) where the $n$ labels of blocks of each member set $S_{d, w}$ constitute $n$ blocks of an orthogonal diallel cross design in $\mathfrak{D}_{0}((m-1) n, n,(m-1) n / 2)$. These designs can be constructed by selecting $(m-1)$ disjoint sets of labels of lines $H_{j}$ from some class $\mathcal{H}$ denoted by $\mathcal{H}=\left\{H_{w}: H_{w}=\left\{h_{n w}, h_{n w+1}, \ldots, h_{n(w+1)-1}\right\} ; 1 \leq h_{n w+j} \neq h_{n w^{\prime}+j^{\prime}} \leq\right.$ $\left.m n ;(w, j) \neq\left(w^{\prime}, j^{\prime}\right), w=0,1,2, \ldots,(m-1)\right\}$ which represents partition of labels of $m n$ lines. In other words, the designs in $\mathfrak{D}_{0 e}^{*}(m n, m n,(m-1) n / 2)$ are nested orthogonal diallel cross designs with maximum trace.

TheOREM 5.1. Let $m=2 t+1, n=2 t^{*}+1$. Consider a randomized block design with $n$ treatments and $m$ blocks. Corresponding to each treatment $u, 0 \leq$ $u \leq n-1$ and its 0 th replication, construct $n$ blocks each of size $(m-1) n / 2$, where uth block is obtained by its block content of the form $\left\{\left(a_{u}^{j}, a_{u}^{m-j}\right): 1 \leq j \leq\right.$ $\left.(m-1) / 2 ;\left(a_{k+u}^{j}, a_{n-k+u}^{j}\right): 1 \leq k \leq(n-1) / 2,1 \leq j \leq m-1\right\}$, where $m-j$ reduced $\bmod (m)$ and $k+u, n-k+u$ reduced $\bmod (n)$. Further, corresponding to each treatment $u, 0 \leq u \leq n-1$ and its lth replication, $1 \leq l \leq m-1$, develop the blocks in the same way as described for construction of design $d_{e}(m, n)$. Then the above series of designs $\left\{d_{e}^{*}(m, n)\right\}$ is optimal in $\mathfrak{D}_{0 e}^{*}(m n, m n,(m-1) n / 2)$. 
Proof. By the construction of a design $d$ belonging to $\mathfrak{D}_{0 e}^{*}(p, b, k)$, it is enough to minimize $\operatorname{tr} C_{d}^{2}$ over $\mathfrak{D}_{0 e}^{*}(m n, m n,(m-1) n / 2)$ as maximum value of $\operatorname{tr} C_{d}$ is attained by any design $d$ in this subclass. At the outset, note that, $C_{d}$ is independent under relabelling of blocks. Therefore, without any loss of generality, one can reduce the search space to the class of designs where designs can be partitioned into $m$ orthogonal diallel cross designs by considering standard form of "class of sets of labels of blocks", viz. $\mathcal{S}=\left\{S_{w}^{*}: S_{w}^{*}=\{n w+1, n w+2, \ldots, n(w+\right.$ $1)\}\}$. Further, note that any design within the reduced space is isomorphic to a design obtained by considering partitioning of lines in standard form $\mathcal{H}^{*}$ where $\mathcal{H}^{*}=\left\{H_{w}^{*}: H_{w}^{*}=\{n w+1, n u+2, \ldots, n(w+1)\} ; w=0,1,2, \ldots,(m-1)\right\}$. This is due to the fact that $\operatorname{tr} C_{d}^{2}$ is independent under permutation of lines. Having said that, it is enough to consider problem of minimizing $\operatorname{tr} C_{d}^{2}$ in the re-reduced class of designs. The designs in this class are nested orthogonal diallel cross designs with maximum trace, where the line vs. block incidence matrices of designs may be defined by partitioned matrix where the sub-matrices are either of the form $\mathbf{1}_{n} \mathbf{1}_{n}^{\prime}$ or $0^{n \times n}$.

Define $m$ classes $\mathcal{A}_{l}$ by $\mathcal{A}_{l}=\left\{A_{l, j}: A_{l, j}=\{j+l, m-j+l\}: 1 \leq j \leq\right.$ $(m-1) / 2\} ; 0 \leq l \leq(m-1)$. Here each class consists of $(m-1) / 2$ sets each of cardinality 2 where elements of sets are indices of disjoint partition of lines represented by $Q_{j}, 0 \leq j \leq(m-1)$ (equivalently, $H_{w}^{*}, 0 \leq w \leq(m-1)$ ). Each of these classes is used to generate crosses of lines for developing nested orthogonal diallel cross designs with maximum trace. Note that $\sum_{i<i^{\prime}} g_{d, i i^{\prime}}=$ $m(m-1) n^{2} / 2<\left(\begin{array}{c}m n \\ 2\end{array}\right)$ and for any design $d, x_{d, i i^{\prime}}$ are either $n$ or 0 according as the lines to $i$ and $i^{\prime}$ belong to same set of labels of lines $H_{w}^{*}$ for some $0 \leq w \leq(m-1)$ or not respectively.

As described in Theorem 4.1, suppose there exists a design $d$ in re-reduced class of designs such that $\max _{\left(i, i^{\prime}\right) \neq\left(j, j^{\prime}\right): i<i^{\prime}, j<j^{\prime}}\left|g_{d, i i^{\prime}}-g_{d, j j^{\prime}}\right|=1$ with $g$ values being at most unity. Note that, this is true for $d=d_{e}(m, n)$ from Remark 4.1 which satisfies $g_{d, i i^{\prime}}=0$ and $g_{d, j j^{\prime}}=1$ iff $x_{d, i i^{\prime}}=n$ and $x_{d, j j^{\prime}}=0$ respectively. Denote the cross of lines with both lines from same set $H_{w}^{*}$ or different set $H_{w}^{*}$ and $H_{w^{\prime}}^{*}$, respectively, by $\left(i, i^{\prime}\right)$ and $\left(j, j^{\prime}\right)$. As Theorem 4.1 only directs us to compare two designs with respect to replication of cross $\left(i, i^{\prime}\right)$, therefore, attempt should be made to further reduce $\operatorname{tr} C_{d}^{2}$ subject to $\max _{\left(i, i^{\prime}\right) \neq\left(j, j^{\prime}\right): i<i^{\prime}, j<j^{\prime}}\left|g_{d, i i^{\prime}}-g_{d, j j^{\prime}}\right|=1$. This is done by further decreasing $\operatorname{tr}\left[G_{d}-(1 / k) X_{d}\right]^{2}$ in equation (4.4) through increasing the replication of crosses involving lines which are from same set $H_{w}^{*}$ by unity, i.e. $g_{d^{*}, i i^{\prime}}=1$ (where $x_{d^{*}, i i^{\prime}}=n$ ) while decreasing the replication of crosses involving lines which are from two different sets $H_{w}^{*}$ and $H_{w^{\prime}}^{*}$ by unity, i.e. $g_{d^{*}, j j^{\prime}}=0$ (where $x_{d^{*}, j j^{\prime}}=0$ ) yielding $\operatorname{tr} C_{d^{*}}^{2} \leq \operatorname{tr} C_{d}^{2}$. Evidently, this claim is true as one may first compare $g$ and $x$ values for crosses $\left(i, i^{\prime}\right)$ and $\left(j, j^{\prime}\right)$ in designs $d$ and $d^{*}$, where $g_{d^{*}, j j^{\prime}}=x_{d^{*}, j j^{\prime}}=0 ; g_{d^{*}, i i^{\prime}}=1, x_{d^{*}, i i^{\prime}} / k=2 /(m-$ 1) while $g_{d, j j^{\prime}}=1, x_{d, j j^{\prime}}=0 ; g_{d, i i^{\prime}}=0, x_{d, i i^{\prime}} / k=2 /(m-1)$. Therefore, $0.5\left\{\operatorname{tr}\left[G_{d^{*}}-(1 / k) X_{d^{*}}\right]^{2}-\operatorname{tr}\left[G_{d}-(1 / k) X_{d}\right]^{2}\right\}=\left(g_{d^{*}, j j^{\prime}}-x_{d^{*}, j j^{\prime}} / k\right)^{2}+\left(g_{d^{*}, i i^{\prime}}-\right.$ $\left.x_{d^{*}, i i^{\prime}} / k\right)^{2}-\left(g_{d, j j^{\prime}}-x_{d, j j^{\prime}} / k\right)^{2}-\left(g_{d, i i^{\prime}}-x_{d, i i^{\prime}} / k\right)^{2} \leq 0$. This reduction can be systematically performed by modifying initial $n$ blocks of design $d_{e}(m, n)$ after 
adding and deleting crosses of the form $\left(i, i^{\prime}\right)$ and $\left(j, j^{\prime}\right)$ respectively. Note that, $\operatorname{tr} C_{d}^{2}$ may be at most decreased by including all the $(m-1) n(n-1) / 2$ crosses of the form $\left(i, i^{\prime}\right)$ in the design, in place of same number of crosses of the form $\left(j, j^{\prime}\right)$. It is not possible to further decrease $\operatorname{tr}\left(C_{d}^{2}\right)$, because number of indices $l$ such that both $w$ and $w^{\prime}\left(1 \leq w<w^{\prime} \leq m-1\right)$ do not belong to any set $A_{l, j}$, $1 \leq j \leq(m-1) / 2$, is zero, and therefore, any inclusion of crosses of the form $\left(i, i^{\prime}\right)$ would lead to some $g$ value to become more than unity, which should not decrease $\operatorname{tr} C_{d}^{2}$ in the class of designs under study. This completes the proof.

Note. The series of designs $\left\{d_{e}^{*}(m, n)\right\}$ can be obtained by following the steps discussed in Remark 4.1 and in the statement of Theorem 5.1. However, computer code is developed in $\mathrm{C}++$ to generate $\left\{d_{e}^{*}(m, n)\right\}$, where $m$ and $n$ are odd integers and included in the Appendix. Here three-dimensional array is used to store the indices of sets of lines which runs iteratively to generate series of designs, and is quite involved.

Remark 5.1. It may be observed that $d_{e}(m, n) \in \mathfrak{D}_{0 e}^{*}(m n, m n,(m-1) n / 2)$. But, using construction method delineated in Theorem 5.1, one may get series of optimal designs $d_{e}^{*}(m, n)$, where $d_{e}^{*}(m, n)>d_{e}(m, n)$; so more efficient series of designs is possible. Since efficiencies of designs $d_{e}(m, n)$ are already found to be near maximum value, viz. unity (see Table 1), therefore efficiencies of designs $d_{e}^{*}(m, n)$ are more near to unity. It may be noted that kind of series of designs $d_{e}(m, n)$ (Example 4.3) is relatively simple to construct whereas $d_{e}^{*}(m, n)$ (Example 5.1) has desirable optimality property but relatively complicated to construct.

Remark 5.2. Let $n$ be finite. Using Remark 4.3, and variance-range inequality, the range for the set of eigen values is at most $\mathrm{O}(\sqrt{m})$ because $\operatorname{tr} C_{d_{e}^{*}}^{2} \leq \operatorname{tr} C_{d_{e}}^{2}$. Observing that $\operatorname{tr} C_{d_{e}^{*}}=\sum_{i=1}^{p-1} \lambda_{i, d_{e}^{*}}=2 b(k-1)=\mathrm{O}\left(m^{2}\right)$, it follows $\lambda_{d_{e}^{*} i} / \lambda_{d_{e}^{*} j} \rightarrow 1$ as $m \rightarrow \infty$ implying the series of designs $d_{e}^{*}(m, n)$ are asymptotically universally optimal with high efficiencies in the context of $A, D, E$-optimality under fixed effects model.

Remark 5.3. From Remark 4.3, and the fact $\operatorname{tr} C_{d_{e}^{*}}^{2} \leq \operatorname{tr} C_{d_{e}}^{2}$, it can be shown that, $\operatorname{eff}\left(d_{e}(m, n)\right) \rightarrow 1$ as $m, n \rightarrow \infty$ which implies the series of designs $d_{e}(m, n)$ are asymptotically optimal and asymptotically MS-optimal under fixed effects model. Finally, Remark 4.5 shows that the series of designs $d_{e}^{*}(m, n)$ are useful for providing a consistent estimator of heritability.

A diagrammatic representation showing relative positions of designs $d_{e}(m, n), d_{e}^{*}(m, n)$ and $\mathfrak{D}(m n, m n,(m-1) n / 2) \supset \mathfrak{D}_{0 e}(m n, m n,(m-1) n / 2) \supset$ $\mathfrak{D}_{0 e}^{*}(m n, m n,(m-1) n / 2)$ is presented in Figure 1. 


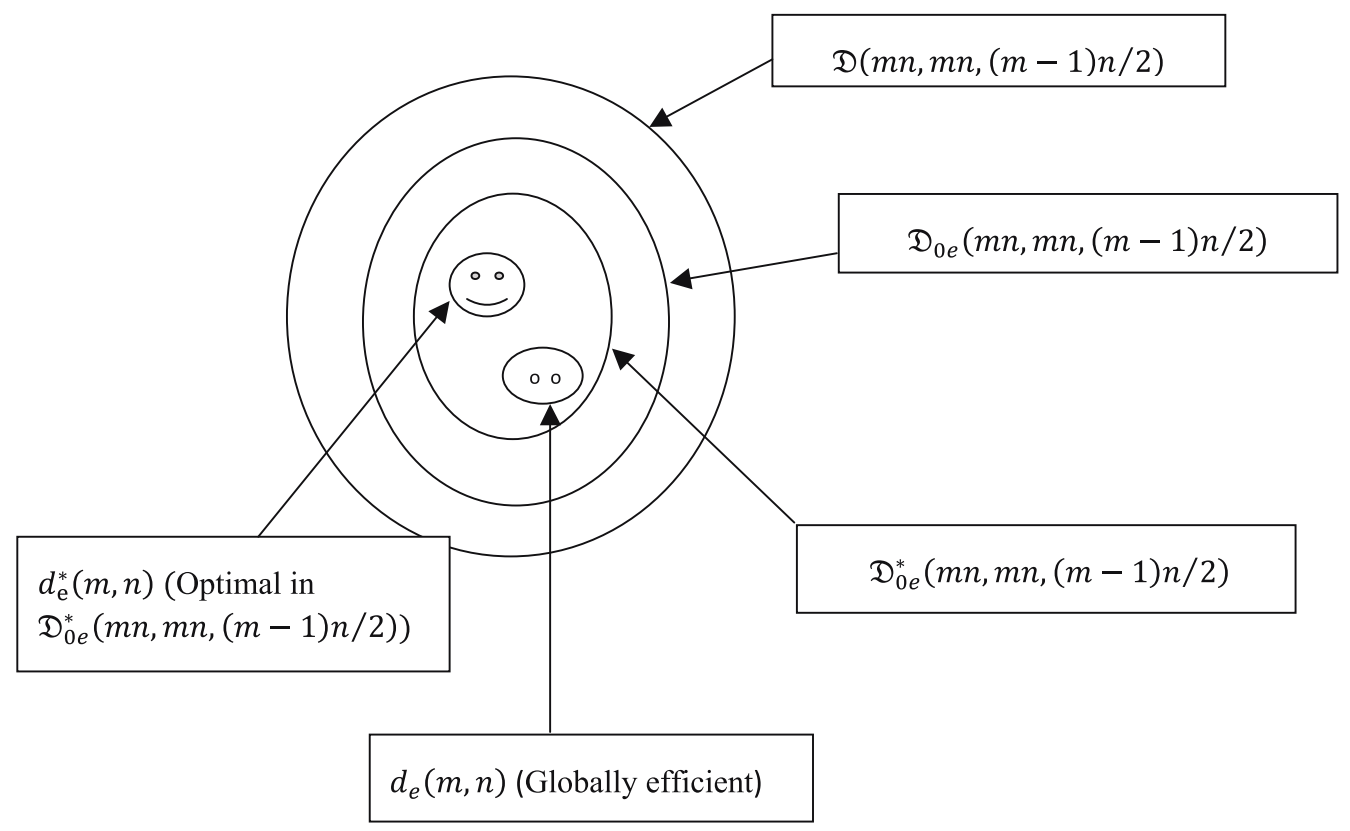

Figure 1. Optimal and Efficient designs with $p=b=m n, k=(m-1) n / 2$.

Example 5.1. Consider the following design with parameters $m=3, n=5$ thereby $p=b=15$, and $k=5$.

\begin{tabular}{lcllll}
\hline Block I & $(6,11)$ & $(7,10)$ & $(8,9)$ & $(12,15)$ & $(13,14)$ \\
Block II & $(7,12)$ & $(6,8)$ & $(9,10)$ & $(11,13)$ & $(14,15)$ \\
Block III & $(8,13)$ & $(7,9)$ & $(6,10)$ & $(12,14)$ & $(11,15)$ \\
Block IV & $(9,14)$ & $(6,7)$ & $(8,10)$ & $(11,12)$ & $(13,15)$ \\
Block V & $(10,15)$ & $(6,9)$ & $(7,8)$ & $(11,14)$ & $(12,13)$ \\
Block VI & $(1,11)$ & $(2,12)$ & $(3,13)$ & $(4,14)$ & $(5,15)$ \\
Block VII & $(1,12)$ & $(2,13)$ & $(3,14)$ & $(4,15)$ & $(5,11)$ \\
Block VIII & $(1,13)$ & $(2,14)$ & $(3,15)$ & $(4,11)$ & $(5,12)$ \\
Block IX & $(1,14)$ & $(2,15)$ & $(3,11)$ & $(4,12)$ & $(5,13)$ \\
Block X & $(1,15)$ & $(2,11)$ & $(3,12)$ & $(4,13)$ & $(5,14)$ \\
Block XI & $(1,6)$ & $(2,7)$ & $(3,8)$ & $(4,9)$ & $(5,10)$ \\
Block XII & $(1,7)$ & $(2,8)$ & $(3,9)$ & $(4,10)$ & $(5,6)$ \\
Block XIII & $(1,8)$ & $(2,9)$ & $(3,10)$ & $(4,6)$ & $(5,7)$ \\
Block XIV & $(1,9)$ & $(2,10)$ & $(3,6)$ & $(4,7)$ & $(5,8)$ \\
Block XV & $(1,10)$ & $(2,6)$ & $(3,7)$ & $(4,8)$ & $(5,9)$ \\
\hline
\end{tabular}

To construct above design, let us first identify 15 lines labeled as $\{1,2, \ldots, 15\}$ by $a_{u}^{j}=5 j+u+1$, i.e. first group of 5 lines viz. $\{1,2, \ldots, 5\}$ are identified 
as $\left\{a_{0}^{0}, a_{1}^{0}, a_{2}^{0}, a_{3}^{0}, a_{4}^{0}\right\}$; second group of 5 lines viz. $\{6,7, \ldots, 10\}$ are identified as $\left\{a_{0}^{1}, a_{1}^{1}, a_{2}^{1}, a_{3}^{1}, a_{4}^{1}\right\}$; followed by third group of 5 lines viz. $\{11,12, \ldots, 15\}$ are identified as $\left\{a_{0}^{2}, a_{1}^{2}, a_{2}^{2}, a_{3}^{2}, a_{4}^{2}\right\}$. As described in Remark 4.1, consider a randomized block design with 5 treatments arranged in 3 blocks which may be symbolically represented as:

\begin{tabular}{llll}
\hline $0_{0}$ & $1_{0}$ & $\cdots$ & $4_{0}$ \\
$0_{1}$ & $1_{1}$ & $\cdots$ & $4_{1}$ \\
$0_{2}$ & $1_{2}$ & & $4_{2}$ \\
\hline
\end{tabular}

The blocks of $d_{e}^{*}(3,5)$ are constructed by following Step (ii) of Remark 4.1, and statement of Theorem 5.1. Finally, the set of 15 blocks each of size 5 are constructed to obtain optimal PDC design in the class $\mathfrak{D}_{0 e}^{*}(15,15,5)$ where each cross appears at most once in the design.

\section{Concluding remarks}

The paper gives two new methods of constructing PDC designs for consistent and unbiased estimation of heritability, which is a nonlinear function of variance components arising out of random effects model of diallel cross experiment. However, there are some other methods of estimation of variance components like maximum likelihood, minimum norm quadratic unbiased estimation. Therefore, attempt would be made to study new unbiased estimator of heritability by reparameterization of variance components so that variance of estimator is minimum for a given design. The variance of estimator could be minimized over a class of optimal design. Further, the random effects model of diallel cross experiment may be extended to include specific combining ability (s.c.a.) effects. To this end, total genetic variance is sum of variances due to g.c.a. and s.c.a., and, accordingly narrow sense heritability is defined by $h^{2}=4 \sigma_{g}^{2} /\left(2 \sigma_{g}^{2}+\sigma_{s}^{2}+\sigma_{e}^{2}\right)$ where $\sigma_{s}^{2}$ denotes variance due to s.c.a. effects (see Falconer (1989)). It is then required to propose estimator of narrow sense heritability and characterize efficient/optimal designs which minimizes the variance of the estimator.

\section{Appendix}

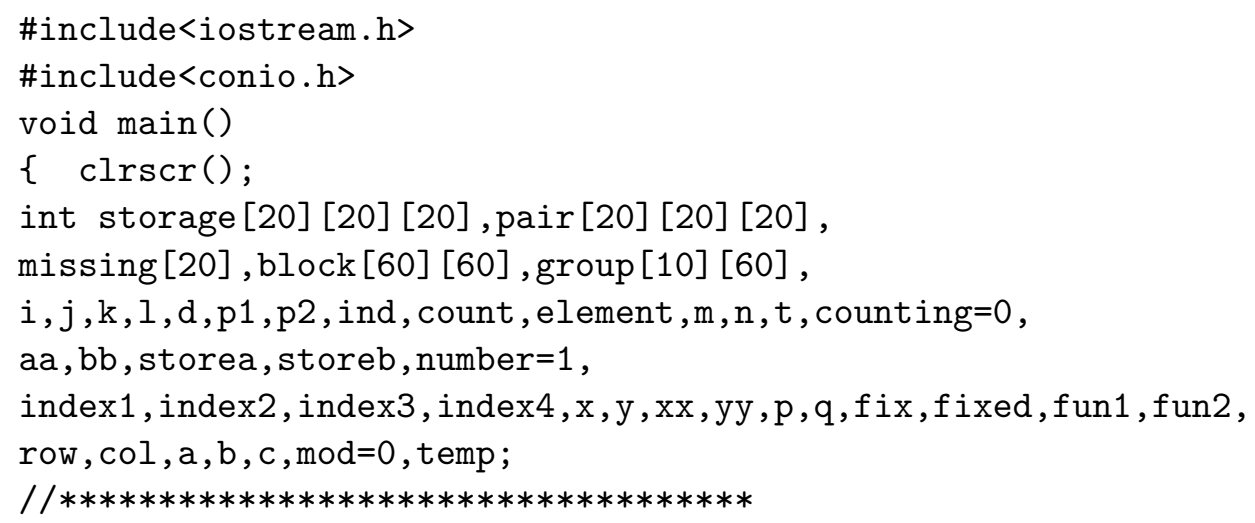




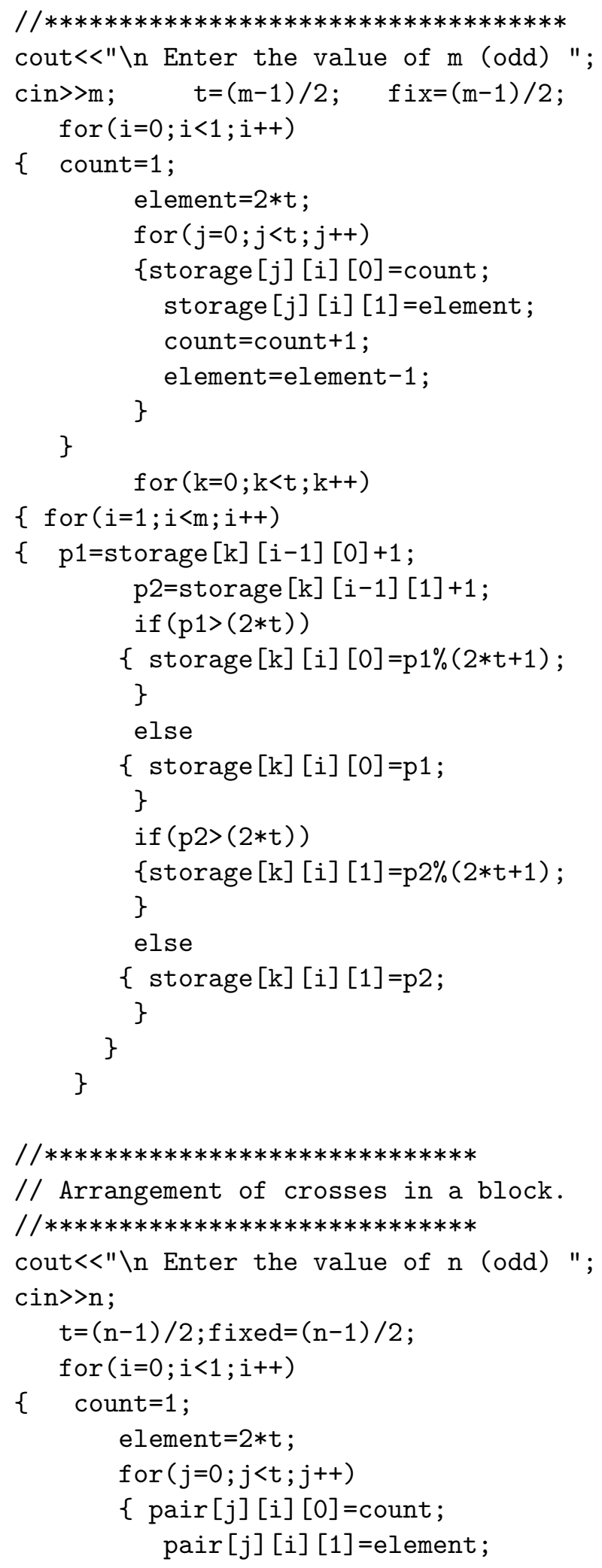

// Arrangement of crosses in a block.

$/ / * * * * * * * * * * * * * * * * * * * * * * * * * * * * *$

cout<<" \n Enter the value of $\mathrm{n}$ (odd) ";

cin $>>\mathrm{n}$;

$\mathrm{t}=(\mathrm{n}-1) / 2 ;$ fixed $=(\mathrm{n}-1) / 2$;

for $(i=0 ; i<1 ; i++)$

$\{$ count $=1$;

element $=2 * t$;

for $(j=0 ; j<t ; j++)$

$\{$ pair $[j][i][0]=$ count ;

pair $[j][i][1]=$ element ; 


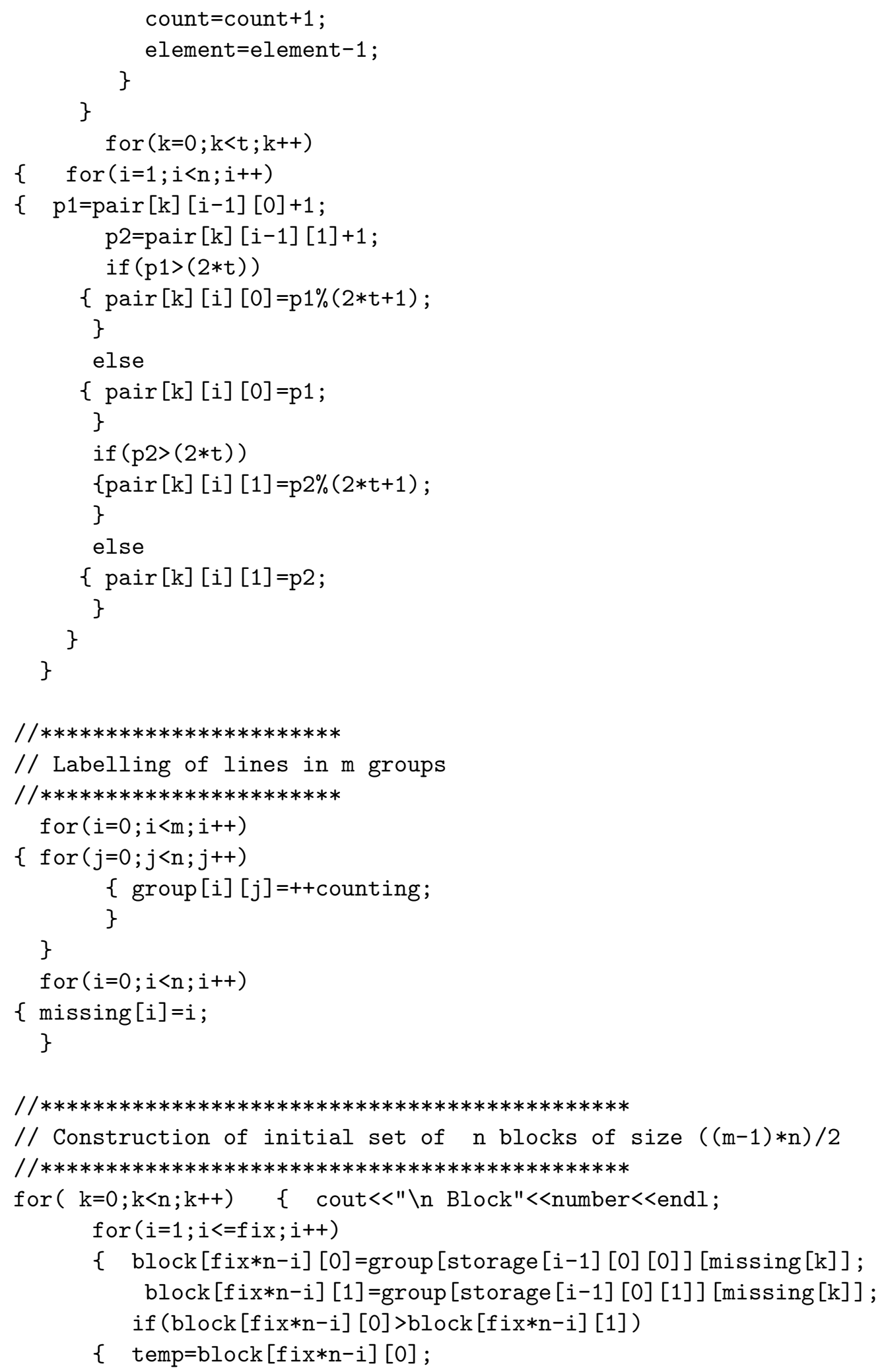




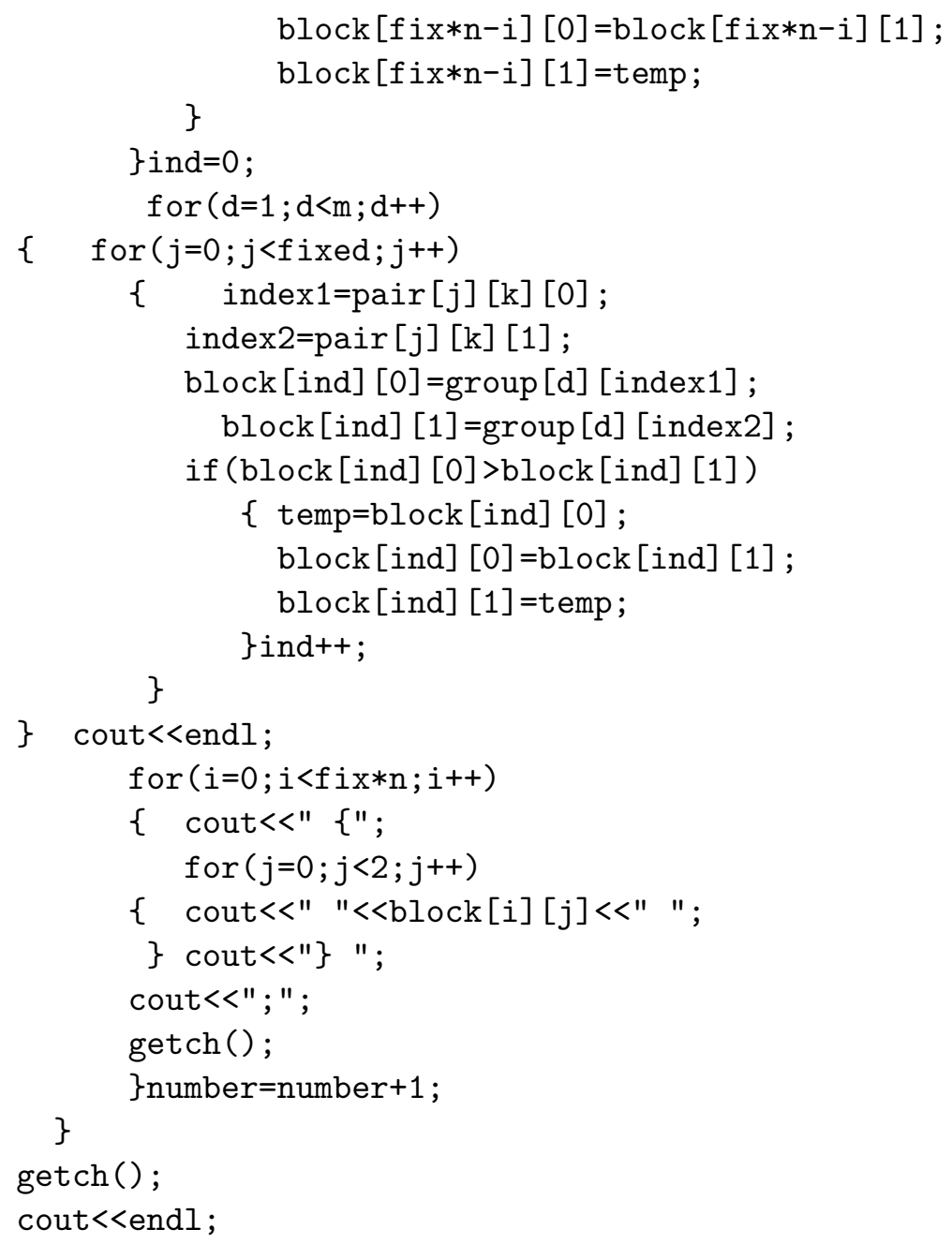

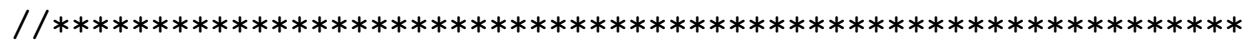

//Construction of (m-1) sets of blocks each containing $\mathrm{n}$ blocks //of size $((\mathrm{m}-1) * \mathrm{n}) / 2$.

$/ / * * * * * * * * * * * * * * * * * * * * * * * * * * * * * * * * * * * * * * * * * * * * * * * * * * * * * * * * * * * * * * * *$

for $(\mathrm{a}=1 ; \mathrm{a}<\mathrm{m} ; \mathrm{a}++)$

$\{$ for $(\mathrm{k}=-1 ; \mathrm{k}<\mathrm{n}-1 ; \mathrm{k}++)$

$\{$ cout $<<" \backslash$ n Block" $<<$ number $<<$ endl;

$\mathrm{q}=\mathrm{k} ; \mathrm{b}=0$;

for $(i=0 ; i<f i x * n ; i++)$

$\{\bmod =i \% n$;

if $(\bmod ==0 \quad \& \& i>0)$

$\{b=b+1$;

\}

$\mathrm{p}=\bmod$;

$\mathrm{q}=\mathrm{q}+1$; 


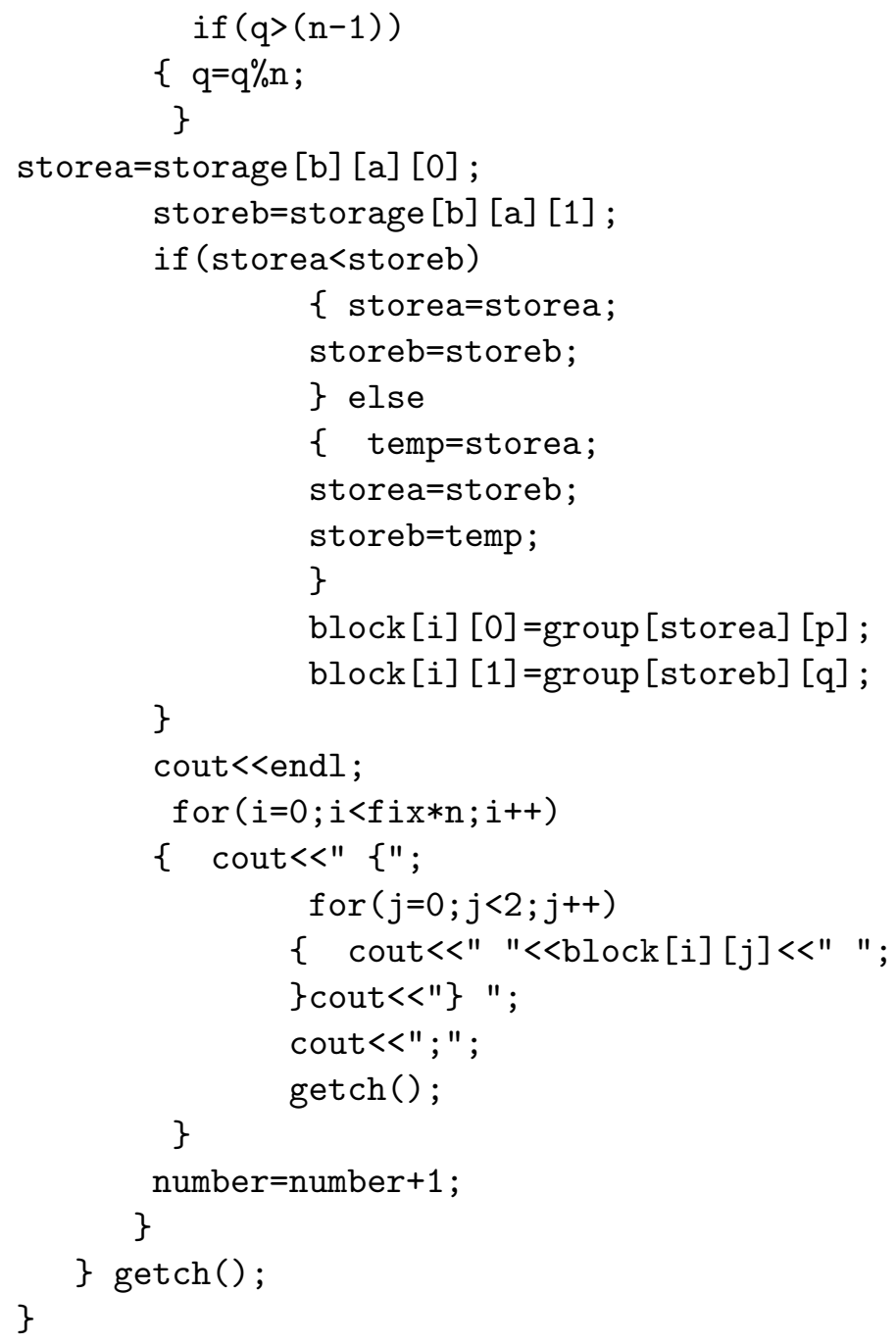

\section{Acknowledgements}

The author is grateful to the three referees and an Associate Editor for their valuable comments, which have greatly improved the overall quality of the article. I also thank Professor Kurata, H., Editor-in-Chief for his cooperation and encouragement, which have been instrumental for making me successful towards acceptance of the paper.

\section{REFERENCES}

Cheng, C. S. (1978). Optimality of certain asymmetric factorial designs, Ann. Statist., 6, 12391261.

Das, A., Dey, A. and Dean, A. M. (1998a). Optimal block designs for diallel cross experiments, Statist. Probab. Lett., 36, 427-436.

Das, A., Dean, A. M. and Gupta, S. (1998b). On optimality of some partial diallel cross designs, Sankhya, 60, 511-524.

Falconer, D. S. (1989). Introduction to Quantitative Genetics, Third Edition, Longman Scientific \& Technical, Essex CM20 2JE, England. 
Ghosh, H. and Das, A. (2003a). Optimal diallel cross designs for estimation of heritability, $J$. Statist. Plann. Infer., 116, 185-196.

Ghosh, H. and Das, A. (2003b). Optimal diallel cross designs for the interval estimation of heredity, Statist. Probab. Lett., 67, 47-55.

Ghosh, H., Das, A. and Midha, C. K. (2005). Optimal design for estimation of ratio of variance components in diallel crosses, Sankhya, 67, 785-794.

Ghosh, H., Das, A. and Midha, C. K. (2006). On some efficient partial diallel cross designs, Statist. Probab. Lett., 76, 1768-1774.

Griffing, B. (1956). Concept of general and specific combining ability in relation to diallel crossing system, Austral. J. Biol. Sci., 9, 463-493.

Gupta, S. and Kageyama, S. (1994). Optimal complete diallel crosses, Biometrika, 81, 420-424.

Gupta, S., Das, A. and Kageyama, S. (1995). Single replicate orthogonal block designs for circulant partial diallel crosses, Commun. Statist.-Th. Meth., 24, 2601-2607.

Hinkelmann, K. (1975). Design of genetical experiments, in A Survey of Statistical Design and Linear Models (ed. J. N. Srivastava), pp. 243-269, Amsterdam, North-Holland.

Kempthorne, O. and Curnow, R. N. (1961). The partial diallel cross, Biometrics, 17, 229-250.

Mukerjee, R. (1997). Optimal partial diallel crosses, Biometrika, 84, 939-948.

Singh, M., Gupta, S. and Parsad, R. (2012). Genetic crosses experiments, in Design and Analysis of Experiments - Special Design and Applications, Vol. 3 (ed. K. Hinkelmann), pp. 1-70, Wiley, New York.

Singh, M. and Hinkelmann, K. (1988). Partial diallel crosses in incomplete blocks, Technical Report No. 88-27, Department of Statistics, Virginia Polytechnique Institute and State University.

Singh, M. and Hinkelmann, K. (1995). Partial diallel crosses in incomplete blocks, Biometrics, 51, 1302-1314. 